\title{
An iterative method for constructing stellar systems models: how far does it work?
}

\author{
Natalia Ya. Sotnikova and Sergei A. Rodionov \\ Saint-Petersburg State University, Saint-Petersburg, Russia \\ email: nsot@astro.spbu.ru
}

\begin{abstract}
We present a new method for constructing equilibrium phase models for stellar systems. Applications of the iterative method include both modelling of observational data and the construction of initial condition for N-body simulations.
\end{abstract}

Keywords. stellar dynamics methods: N -body simulations galaxies: kinematics and dynamics galaxies: structure

The aim of the iterative method (IM) is to construct an equilibrium N-body model with prescribed parameters, or constraints. Setting a given mass distribution and almost arbitrary velocities of particles, we start the iterative procedure, by letting the system go through a sequence of self-consistent evolutionary steps of short duration (iterations). At the end of each step, and before the new step is started, we transfer the new velocity distribution from a bit evolved system to a system with the initial density distribution. At this stage we need to correct individual particle velocities in accordance with imposed kinematic constraints (see details in Rodionov et al. 2009). We stop iterations when the velocity distribution ceases to change, which implies that the system has reached equilibrium.

We managed to construct equilibrium systems of various types - from spherical to triaxial, from one-component to multi-component, from isotropic to anisotropic (Rodionov \& Sotnikova (2006); Sotnikova \& Rodionov (2008); Rodionov et al. (2009)). Successful reconstruction of the distribution function of a model disc galaxy from its line-of-sight kinematics encouraged us to use the IM to derive the 3D kinematics of edge-on galaxies from observational data. Now we have all IR photometric parameters (for a bulge and a disc) of an edge-on galaxy NGC 4111 and obtained stellar LSVD of this galaxy at the 6-m telescope. Preliminary interpretation of kinematic and photometric observations of this galaxy in terms of its $3 \mathrm{D}$ structure and $3 \mathrm{D}$ velocity distribution showed that the IM may be very powerful method to reconstruct phase-space models of real galaxies (Sotnikova et al., 2010, in preparation).

\section{Acknowledgements}

This work was supported by the Russian Foundation for Basic Research (grant 09-0200968) and by a grant from President of the RF for support of Leading Scientific Schools (grant NSh-1318.2008.02).

\section{References}

Rodionov, S. A. \& Sotnikova, N. Ya. 2006, Astron. Rep. 50, 983

Sotnikova N. Ya. \& Rodionov, S. A. 2008, Astron. Lett. 34, 664

Rodionov, S. A., Athanassoula, E., \& Sotnikova, N. Ya. 2009, MNRAS 392, 904 